\title{
Action video game playing is associated with improved visual sensitivity, but not alterations in visual sensory memory
}

\author{
L. Gregory Appelbaum • Matthew S. Cain • \\ Elise F. Darling • Stephen R. Mitroff
}

Published online: 25 May 2013

(C) Psychonomic Society, Inc. 2013

\begin{abstract}
Action video game playing has been experimentally linked to a number of perceptual and cognitive improvements. These benefits are captured through a wide range of psychometric tasks and have led to the proposition that action video game experience may promote the ability to extract statistical evidence from sensory stimuli. Such an advantage could arise from a number of possible mechanisms: improvements in visual sensitivity, enhancements in the capacity or duration for which information is retained in visual memory, or higher-level strategic use of information for decision making. The present study measured the capacity and time course of visual sensory memory using a partial report performance task as a means to distinguish between these three possible mechanisms. Sensitivity measures and parameter estimates that describe sensory memory capacity and the rate of memory decay were compared between individuals who reported high evels and low levels of action video game experience. Our results revealed a uniform increase in partial report accuracy at all stimulus-tocue delays for action video game players but no difference in the rate or time course of the memory decay. The present findings suggest that action video game playing may be related to enhancements in the initial sensitivity to visual stimuli, but not to a greater retention of information in iconic memory buffers.
\end{abstract}

\footnotetext{
L. G. Appelbaum $(\bowtie) \cdot$ E. F. Darling $\cdot$ S. R. Mitroff

Center for Cognitive Neuroscience, Duke University,

Durham, NC 27708, USA

e-mail: greg@duke.edu

L. G. Appelbaum

Department of Psychiatry and Behavioral Sciences,

Duke University, Durham, NC, USA
}

\section{S. Cain}

Department of Cognitive, Linguistic \& Psychological Sciences, Brown University, Providence, RI, USA

E. F. Darling $\cdot$ S. R. Mitroff

Department of Psychology \& Neuroscience, Duke University,

Durham, NC, USA
Keywords Visual learning · Plasticity · Iconic memory

\section{Introduction}

Individuals with extensive experience with action video games exhibit enhanced performance in a number of sensory, perceptual, and attentional domains, as compared with individuals with limited-to-no action video game experience. When compared with non-video game players (NVGPs), action video game players (AVGPs) tend to respond more rapidly (Castel, Pratt, \& Drummond, 2005; Dye, Green, \& Bavelier, 2009; Orosy-Filders \& Allan, 1989; Yuji, 1996), can simultaneously apprehend and track a greater number of items (Green \& Bavelier, 2006; Trick, Jaspers-Fayer, \& Sethi, 2005), possess improved spatial (Boot, Kramer, Simons, Fabiani, \& Gratton, 2008; Feng, Spence, \& Pratt, 2007; Green \& Bavelier, 2003, 2006, 2007; Okagaki \& Frensch, 1994; Quaiser-Pohl, Geiser, \& Lehmann, 2006; Terlecki \& Newcombe, 2005) and temporal (Donohue, Woldorff, \& Mitroff, 2010; Green \& Bavelier, 2003; Li, Polat, Makous, \& Bavelier, 2009; West, Stevens, Pun, \& Pratt, 2008) abilities, and demonstrate better task-switching (Boot et al., 2008; Cain, Landau, \& Shimamura, 2012; Colzato, van Leeuwen, van den Wildenberg, \& Hommel, 2010; Karle, Watter, \& Shedden, 2010; Strobach, Frensch, \& Schubert, 2012) and strategic (Chisholm, Hickey, Theeuwes, \& Kingstone, 2010; Clark, Fleck, \& Mitroff, 2011) skills. Collectively, these behavioral benefits extend well beyond the specific context of the activities carried out in the games themselves and have spawned a line of experimental research aimed at identifying the specific capacities and mechanisms that are altered as a result of action video game experience.

Action video game play, for example, has been widely linked to enhancements in the spatial and temporal resolution of vision. As compared with NVGPs, AVGPs have lower contrast sensitivity thresholds and are therefore able to detect smaller changes in the levels of gray in grating patterns ( $\mathrm{Li}$ et 
al., 2009). Similarly, AVGPs have improved target detection abilities that span across the useful field of view (Green \& Bavelier, 2006; Feng et al., 2007) and exist for target stimuli, such as abrupt changes in motion direction (West et al., 2008). In addition to improved visual-spatial abilities, AVGPs have been shown to generally process visual information faster (Dye et al., 2009; Green \& Bavelier, 2012; Green, Pouget, \& Bavelier, 2010) and to exhibit a superior capacity to instantaneously extract information from visual displays (Green \& Bavelier, 2006). Of note among these findings, it has been demonstrated that relative to NVGPs, AVGPs are able to enumerate rapidly presented items more accurately when displays contain more than four items (i.e., beyond the typical subitizing range; Mandler \& Shebo, 1982). These, and other, findings suggest that action gaming experience may modulate the earliest aspects of visual sensory processing; however, the specific mechanisms enabling these enhancements is still an area of active debate.

Beyond these types of findings related to perceptual abilities, an interesting juxtaposition has begun to emerge in the findings relating AVGP experience to attentional abilities. Namely, while AVGP benefits have been observed in many facets of top-down "executive" attentional control, such as selective attention (Chisholm et al., 2010; Chisholm \& Kingstone, 2012; West et al., 2008), divided attention (Dye et al., 2009; Green \& Bavelier, 2006), and sustained attention (Clark et al., 2011), bottom-up attention driven by exogenous cues does not appear to differ between AVGPs and NVGPs (Castel et al., 2005; Dye, Green, \& Bavelier, 2009; Hubert-Wallander, Green, Sugarman, \& Bavelier, 2011; Wu et al., 2012). While some debate still exists as to the reliability of these findings (Cain et al., 2012; Irons, Remington, \& McLean, 2011), a consensus is emerging that action video game experience is associated not only with an enhancement of attentional resources, but also with a more efficient allocation of these resources (Bavelier, Green, Pouget, \& Schrater, 2012).

While findings such as those described above point to enhancements in sensory and attentional processing for AVGPs, the degree to which action video games might influence the retention of information in the various stages of memory is less clear. Previous studies have generally found modest enhancements in short-term memory tasks but little in the way of longer-term memory benefits. For example, AVGPs typically show better performance on $N$-back tasks (Boot et al., 2008; Colzato, van den Wildenberg, Zmigrod, \& Hommel, 2013), better change detection abilities (Boot et al., 2008; Clark et al., 2011), and more accurate memory representations of objects (Sungur \& Boduroglu, 2012) but no enhancements in short-term verbal recall (Cain et al., 2012) or in the capacity of visual short-term memory (Wilms, Petersen, \& Vangkilde, 2013) and minimal-or possibly detrimental-effects in tasks that require long-term memory access. No previous studies, however, have sought to characterize the time course of visual sensory memory (VSM) as a function of action gaming experience. As such, it remains unknown whether AVGPs and NVGPs differ in their abilities to retain and utilize information in early memory buffers.

In light of the diverse behavioral benefits reported above, recent computational accounts of video-game-related learning have begun to provide a unifying framework under which such advantages may arise. In particular, it has been suggested that such performance benefits can result from the more efficient use of sensory information in order to better compute the probability that various choices are correct, given the evidence that is present (Bavelier et al., 2012; Green \& Bavelier, 2012; Green et al., 2010). By applying a neural model of visual motion processing to behavioral motion discrimination data collected from AVGPs and NVGPs, it was found that the pattern of behavioral differences between the two groups was primarily captured through a single parameter change - the connectivity between the sensory and integration layers of the model (Green et al., 2010). This increase in connectivity was interpreted as AVGPs being able to accumulate information toward a decision more quickly over time than NVGPs. This enhanced ability has been referred to as improved probabilistic inference.

Mechanistically, improved probabilistic inference could arise from any of several possible differences in the visualinformation-processing stream, and here we examine three candidates. First, these enhancements might result from an increase in perceptual sensitivity wherein more information is available to AVGPs as they are making a decision about how to act or respond to a stimulus. Second, initial visual sensitivity may remain unchanged, but sensory information may be retained longer in visual memory, thereby mitigating the natural decay of information in memory buffers as evidence is accumulated toward a particular decision choice. Third, there may be no differences in the initial sensitivity to visual information or in the retention of this information in rapidly decaying sensory memory buffers, but rather decision processes may be enhanced such that AVGPs are able to more quickly or reliably "read out" perceptual information (Gold \& Ding, 2012), leading to improved performance.

To distinguish between these three possible mechanisms, we measured the capacity and time course of VSM (also called iconic memory; Neisser, 1967) for individuals who scored either high or low on a self-report video game experience questionnaire. Individuals participated in a partial report memory task (Sperling, 1960) in which they were cued to report the identity of an item from a larger display that was briefly presented but disappeared before the cue. Because participants did not know which item from this set would be cued for recall, performance on this task can be regarded as a random sample of the memory for the entire display. As such, this task provides a continuous measure of memory retention 
at delays spanning from sensory (Coltheart, 1980) through short-term (Long, 1980) memory durations. Importantly, the time course and capacity of memory captured in the partial report task can be quantified through the psychometric modeling of three parameters that map onto the three possible mechanisms under consideration here: $a_{1}$ reflects the initial visual availability of stimulus information; $\tau$ indicates the decay rate and duration of sensory memory, thereby determining how much information is available for transfer into shortterm memory; and $a_{0}$ captures the amount of information that is retained in short-term memory (Bradley \& Pearson, 2012; Graziano \& Sigman, 2008; Kuhbandner, Spitzer, \& Pekrun, 2011; Lu, Neuse, Madigan, \& Dosher, 2005). By contrasting the persistence of visual information across these varying spans of memory retention, we are able to infer which mechanism(s) may underlie the broad differences observed between AVGPs and NVGPs.

\section{Method}

\section{Participants}

The present study involved 130 individuals who participated in a larger research endeavor conducted in the Duke University Visual Cognition Lab. As part of the research protocol, individuals participated in two experimental phases: an individual-differences assessment phase and a behavioraltesting phase. Participants were recruited through campus advertisements and the Psychology Department participant pool and received either $\$ 10$ per hour or course credit in compensation. An advantage of this two-phase design is that it reduces expectation biases (Boot, Blakely, \& Simons, 2011), since the participants complete dozens of assessments without any being more notable than the others. As such, the participants in the present study had no reason to expect that their video gaming experiences (or lack thereof) were relevant to their experimental participation. All individuals were 18 or older (18-53 years, $M=21.45$ years) and voluntarily participated under an experimental protocol approved by Duke University's Institutional Review Board. From the initial sample of 130 participants, 5 were removed as outliers on the basis of their behavioral performance (VSM parameter estimates; see below) falling more than 3 standard deviations beyond the mean. ${ }^{1}$ This left 125 participants in the final analysis (52 male, 73 female).

\section{Individual-differences assessments}

On the first day of a 2-day experiment, participants completed self-report assessments that measured characteristics across a

\footnotetext{
${ }^{1}$ Among the 5 excluded outliers, 1 would have been categorized as an AVGP, and 1 would have been an NVGP.
}

broad scope of demographic, physiological, clinical, psychological, and pastime-preference information (for more detail on these measures, see Appelbaum et al., 2012; Donohue, Darling, \& Mitroff, 2012). As part of a self-report video gaming experiences questionnaire, the participants were asked to rate their perceived level of expertise on each of eight different video game genres via 7-point $(0-6,6$ being the highest) Likert scale expertise ratings. Participants were defined as AVGPs if they rated their expertise on "action/platforming" or "first person shooter" games as greater than or equal to 5. Individuals were categorized as NVGPs if they rated their expertise on "action/platforming" and "first person shooter" games as 0 . These criteria yielded 31 AVGPs (26 male and 5 female) and 36 NVGPs (6 male and 30 female). These groups did not differ in mean age $($ AVGPs $=20.84$, NVGPs $=20.64), t=0.283, p>.77$, and gender differences are explored further in the Results section, below.

\section{Behavioral testing}

On the second day, all participants performed a VSM task. This task was a modified version of the task used by Lu et al. (2005) and was identical to the task used by Appelbaum, Cain, Schroeder, Darling, and Mitroff (2012). In this experiment, visual stimuli were viewed by the participants from an approximate distance of $57 \mathrm{~cm}$ in a dimly lit room. Each trial began with a black fixation cross at the center of a gray $\left(30 \mathrm{~cd} / \mathrm{m}^{2}\right)$ background. After $400 \mathrm{~ms}$, eight black uppercase letters (each $1.3^{\circ} \times 1.3^{\circ}$ ) appeared arranged on an imaginary circle $\left(3.50^{\circ}\right.$ radius $)$ around fixation. The letters were drawn randomly, with replacement, from the set $\mathrm{D}, \mathrm{F}, \mathrm{J}, \mathrm{K}$, with the constraint that no neighboring letters were the same. After $105 \mathrm{~ms}$, the letter display was replaced by a fixation cross, and then a red line ( $1^{\circ}$ in length, with a circle at the end) appeared after a variable delay, or interstimulus interval (ISI), of $13,40,80,160,320,640,1,280$, or $2,560 \mathrm{~ms}$. The line pointed randomly to one of the eight letter locations and remained visible until response. Participants were to report the identity of the letter at the cued location using the corresponding key on a standard keyboard.

VSM performance was assessed by first computing the mean percent correct for each ISI. Percent correct were converted from four-alternative forced identification to $d^{\prime}$ (a measure of sensitivity; Wickens, 2001). Next, to characterize the temporal properties of iconic memory, a one-phase exponential-decay function was fit to the $d^{\prime}$ data using bestfit least squares method (Bradley \& Pearson, 2012; Lu et al., 2005). This yielded three parameters; $a_{1}$, the fast-decaying sensitivity that reflects the initial visual availability of stimulus information; $\tau$, the time constant of the fast-decay sensitivity that represents the duration of iconic memory; and $a_{0}$, the sensitivity at long delays that reflects the amount of information transferred into short-term memory without the benefit of 


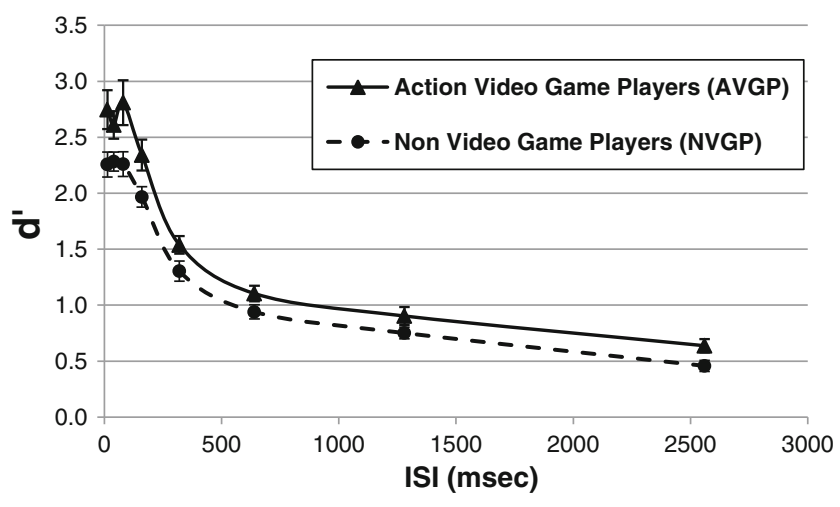

Fig. $1 d^{\prime}$-transformed iconic memory curves for the 31 AVGP (solid) and 36 NVGP (dashed) participants. Error bars indicate $\pm 1 S E M$

cuing (see Lu et al., 2005). The goodness of fit between the observed data and the values predicted by the exponentialdecay functions were good (AVGP, $R=.91$; NVGP, $R=.98$ ) and are in line with other reported studies (Bradley \& Pearson, 2012). Statistical assessment of VSM performance for the AVGP and NVGP groups was accomplished using mixedmodel analyses of variance, (ANOVAs), with nonsphericity correction of the degrees of freedom (Greenhouse-Geisser algorithm) where necessary, or using equal variance assumed $t$-tests and reporting two-tailed $p$-values.

\section{Results}

Participants produced typical memory decay functions with high accuracy at short ISIs and with performance falling to near chance at the longest ISI. Comparisons between the $d^{\prime}$ transformed iconic memory curves for the AVGPs (solid) and NVGPs (dashed) are shown in Fig. 1. Across all ISIs, the AVGPs produced higher $d^{\prime}$ values than did the NVGPs, and these differences reached two-tailed statistical significance at all but three of the ISIs (Table 1). A mixed-model ANOVA performed on the $d^{\prime}$ data with a within-participants factor of ISI (eight levels) and a between-groups factor of action video game playing status (AVGP vs. NVGP) revealed significant within-participants main effects of ISI, $F(4.65,302.18)=168.86, p<.001$, and a significant between-group main effect, $F(1,65)=11.22, p=.001$. Parameter estimates (Fig. 2) were significantly higher for
AVGPs than for NVGPs for both $a_{1}, t=3.41, p=.01$, and $a_{0}, t=2.25, p=.028$, although there was no between-groups effect for the $\tau$ parameter, $t=-0.418, p=.67$.

Are AVGP/NVGP differences driven by gender?

As has commonly been observed in other studies (Boot et al., 2008; Cain et al., 2012; Lucas \& Sherry, 2004), our AVGP group contained more males $(N=26)$ than females $(N=5)$, while the NVGP group had more females $(N=30)$ than males $(N=6)$. To assess whether the observed group differences in VSM performance were merely the result of gender disparities between the groups, two auxiliary analyses were performed. First, the three parameter estimates were compared between all male $(N=52)$ and female $(N=73)$ participants in the full sample of 125 participants, regardless of video game experience. There were no significant differences between the gender groups (Table 2A), indicating that, across the entire sample, males and females had similar VSM performance. Second, we compared the three parameter estimates for all males $(N=20)$ and all females $(N=38)$ who did not meet the criteria to be classified as AVGPs or NVGPs. That is, for this second, additional analysis, we specifically focused on the 58 participants who had too little action video game experience to be classified as an AVGP but too much to be an NVGP. While females in this group tended to have slightly higher parameter estimates, there were no significant differences for any of the three parameters (Table 2B). These auxiliary analyses argue against the notion that the observed AVGP versus NVGP effects were driven by gender differences, since the AVGP sample had higher parameter estimates yet was dominated by males.

\section{Discussion}

In light of the diverse behavioral and computational findings linked to action video games, the present study explored whether action video game experience is associated with changes in the initial sensitivity, time course, and/or capacity of visual information processing. For this purpose, we employed a modified partial report task (Sperling, 1960) that provides a continuous measure of memory retention

Table $1 d^{\prime}$ values and two-tailed paired comparison results contrasting action video game players (AVGPs) and non-video game players (NVGPs) at each of the eight interstimulus intervals

\begin{tabular}{lrrrrrrrr}
\hline ISI & $13 \mathrm{~ms}$ & $40 \mathrm{~ms}$ & $80 \mathrm{~ms}$ & $160 \mathrm{~ms}$ & $320 \mathrm{~ms}$ & $640 \mathrm{~ms}$ & $1,280 \mathrm{~ms}$ & $2,560 \mathrm{~ms}$ \\
\hline AVGP & 2.75 & 2.61 & 2.81 & 2.34 & 1.54 & 1.10 & 0.90 & 0.64 \\
NVGP & 2.26 & 2.28 & 2.26 & 1.97 & 1.30 & 0.94 & 0.75 \\
$p$-value (two-tailed) & .02 & .03 & .02 & .03 & .06 & .09 & .11 & .02 \\
\hline
\end{tabular}




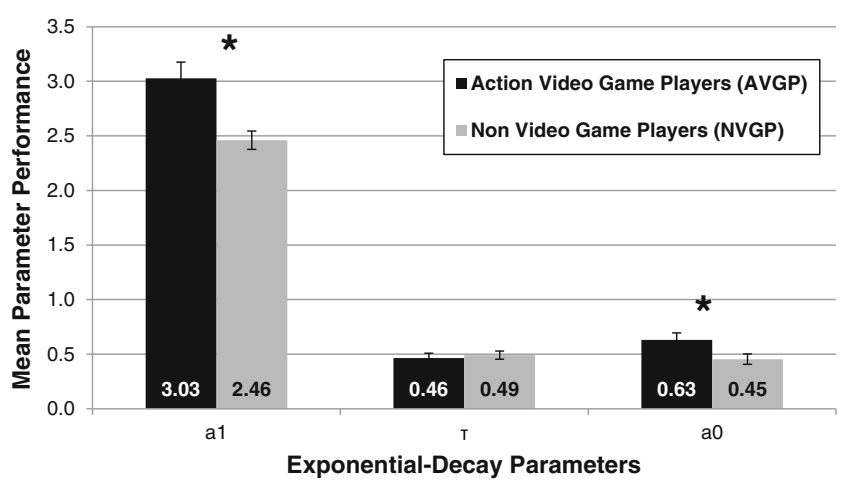

Fig. 2 Parameters estimates were significantly higher for AVGPs (black) than for NVGPs (gray) for $a_{0}$ and $a_{1}$, although no difference was present between the two groups for the $\tau$ parameter. Error bars indicate \pm 1 SEM. "*” indicates two-tailed $p<.05$

by cuing participants at various delays to report the identity of a single item from a larger, briefly presented display. In this manner, the partial report task can be used to quantify the time course and capacity of memory retention, particularly as it relates to the initial sensitivity to stimuli immediately available for reporting, as well as the decay of information in sensory memory that occurs between the initial stimulation and subsequent cue.

Memory performance was markedly higher for AVGPs than for NVGPs, and this benefit was reflected by a relatively uniform increase across all stimulus-to-cue delays (ISIs). This vertical shift in the iconic memory curves, combined with the lack of difference in the $\tau$ parameter, suggests that while AVGPs have a uniformly greater capacity for VSM, there is no difference in the rate of memory decay for the two groups. In the Introduction, we presented three mechanistic accounts of how probabilistic inference could arise: (1) increases in initial perceptual sensitivity, (2) longer retention in iconic memory buffers, or (3) enhanced "readout" of perceptual information into working memory. The present results suggest that action gaming experience is related to differences in initial perceptual sensitivity and may not relate to changes in the duration or persistence of visual information in iconic memory

Table 2 Parameter estimates and two-tailed $p$-values comparing (A) all males and all females in the sample and (B) all males and all females who did not meet the criteria to be classified into either the action video game player or non-video game player group

\begin{tabular}{lccc}
\hline A) & $a_{1}$ & $\tau$ & $a_{0}$ \\
Males $(N=52)$ & 2.84 & 0.48 & 0.56 \\
Females $(N=73)$ & 2.69 & 0.46 & 0.55 \\
$p$-value (two-tailed) & .30 & .71 & .83 \\
$(\mathrm{~B})$ & $a_{1}$ & $\tau$ & $a_{0}$ \\
Males $(N=20)$ & 2.73 & 0.52 & 0.50 \\
Females $(N=38)$ & 2.82 & 0.42 & 0.62 \\
$p$-value (two-tailed) & .73 & .17 & .18 \\
\hline
\end{tabular}

or the ability to move information from iconic to working memory. Importantly, the improved initial sensitivity suggests that more information is available in the sensory memory buffer for AVGPs and, therefore, these individuals will be able to accumulate information toward a decision more quickly over time. As such, the present results provide a mechanistic account for the proposal by Bavelier and colleagues (Bavelier et al., 2012; Green et al., 2010) that AVGPs benefit from improved probabilistic inference wherein increases in neural connectivity are subserved by greater initial availability of information in the processing stream, rather than the prolonged persistence of this information.

The present results reveal a clear group difference between AVGPs and NVGPs in visual sensitivity and suggest that these differences are not due to simple gender differences. However, these differences are based upon a cross-sectional sample of individuals, and future work will be needed to examine the causal nature of these effects. A training study wherein NVGPs are tested on a partial report task before and after an intervention (i.e., either playing action video games or engaging in a matched control task) will allow for distinguishing between a preexisting explanation wherein those with heightened sensitivity self-select to play action video games and a learning explanation wherein the action video game exposure enhances sensitivity (for discussions of causal influences in video game studies, see Boot et al., 2011; Kristjansson, 2013). Regardless of such causal factors, the present results inform both the video game literature and the visual memory literature.

For the video game literature, this study provides a mechanistic explanation for the probabilistic inference theory proposed by Bavelier and colleagues (Bavelier et al., 2012; Green et al., 2010). Decisions about the properties of visual stimuli have been shown to involve the accumulation of noisy sensory information over time until an information criterion has been attained and a decision can be made (Gold \& Shadlen, 2007; Ratcliff \& Smith, 2004). The sensory information retained in iconic memory is accessible for several hundred milliseconds following the disappearance of a stimulus and is thought to be a primary source from which such evidence is accumulated (Nikolić, Häusler, Singer, \& Maass, 2009). The present results suggest that decision making in AVGPs might benefit from the increased availability of information in these iconic memory buffers, but without the added contribution of increased persistence of information in these buffers. However, more work will be needed before we can fully understand how such differences explicitly manifest into decision mechanisms.

For the visual memory literature, the present data speak to the potential influences of experience on iconic memory. While iconic memory has historically been considered a preattentive store of visual information (Neisser, 1967), recent studies have indicated that iconic memory consolidation shares similar neural mechanisms with volitional attention (Ruff, Kristjansson, \& Driver, 2007) and may, in fact, be 
dependent on attentional resources(Persuh, Genzer, \& Melara, 2012). In particular, when placed under demanding dual-task conditions, iconic memory consolidation is impaired such that individuals maintain only gross representations of visual scenes, fail to properly categorize features, and often fail to detect perceptual changes (Persuh et al., 2012). While the present experiment was a single task, a possible interpretation of the present results is that AVGPs benefit from action-gaming-related top-down attentional enhancements, which could produce greater sensitivity even in relatively undemanding visual environments. In fact, such top-down attentional enhancements have been observed experimentally in a number of related contexts (Dye et al., 2009; Green \& Bavelier, 2006; Chisholm et al., 2010; Chisholm \& Kingstone, 2012; Clark et al., 2011; West et al., 2008). Future work in which cognitive load is manipulated specifically in the context of VSM performance may help to support this interpretation.

Acknowledgments This research was funded by grants to S.R.M. from Nike SST, Nike Inc., and the Army Research Office (\#54528LS) and through a subcontract with the Institute for Homeland Security Solutions, a research consortium sponsored by the Resilient Systems Division in the Department of Homeland Security (DHS) through Contract No. HSHQDC-08-C-00100. Support for L.G.A. was provided through DARPA grant D12AP00025-002. Any opinions, findings, and conclusions or recommendations expressed in this material are those of the authors and do not necessarily reflect the official policy or position of DHS or of the U.S. government. The study is approved for public release. We would like to thank Mr. Ken Roberts for providing statistical help and Dr. Tarik Bel-Bahar for his thoughtful comments on the manuscript.

\section{References}

Appelbaum, L. G., Cain, M. S., Darling, E. F., Stanton, S. J., Nguyen, M. T., \& Mitroff, S. R. (2012). What is the identity of a sports spectator? Personality and Individual Differences, 52, 422-427.

Appelbaum, L. G., Cain, M. S., Schroeder, J. E., Darling, E. F., \& Mitroff, S. R. (2012). Stroboscopic visual training improves information encoding in short-term memory. Attention, Perception \& Psychophysics.

Bavelier, D., Green, C. S., Pouget, A., \& Schrater, P. (2012). Brain plasticity through the life span: Learning to learn and action video games. Annual Review of Neuroscience, 35, 391-416.

Boot, W. R., Blakely, D. P., \& Simons, D. J. (2011). Do action video games improve perception and cognition? Frontiers in Psychology, 2, 226.

Boot, W. R., Kramer, A. F., Simons, D. J., Fabiani, M., \& Gratton, G. (2008). The effects of video game playing on attention, memory, and executive control. Acta Psychologica, 129(3), 387-398.

Bradley, C., \& Pearson, J. (2012). The sensory components of highcapacity iconic memory and visual working memory. Frontiers in Psychology, 3, 355.

Cain, M. S., Landau, A. N., \& Shimamura, A. P. (2012). Action video game experience reduces the cost of switching tasks. Attention, Perception, \& Psychophysics, 74(4), 641-647.

Castel, A. D., Pratt, J., \& Drummond, E. (2005). The effects of action video game experience on the time course of inhibition of return and the efficiency of visual search. Acta Psychologica, 119(2), 217-230.

Chisholm, J. D., Hickey, C., Theeuwes, J., \& Kingstone, A. (2010). Reduced attentional capture in action video game players. Attention, Perception, \& Psychophysics, 72(3), 667-671.

Chisholm, J. D., \& Kingstone, A. (2012). Improved top-down control reduces oculomotor capture: The case of action video game players. Attention, Perception, \& Psychophysics, 74(2), 257-262.

Clark, K., Fleck, M. S., \& Mitroff, S. R. (2011). Enhanced change detection performance reveals improved strategy use in avid action video game players. Acta Psychologica, 136(1), 67-72.

Coltheart, M. (1980). Iconic memory and visible persistence. Perception \& Psychophysics, 27(3), 183-228.

Colzato, L. S., van den Wildenberg, W. P., Zmigrod, S., \& Hommel, B. (2013). Action video gaming and cognitive control: Playing first person shooter games is associated with improvement in working memory but not action inhibition. Psychological Research, 77, 234-239.

Colzato, L. S., van Leeuwen, P. J. A., van den Wildenberg, W., \& Hommel, B. (2010). DOOM'd to switch: Superior cognitive flexibility in players of first person shooter games. Frontiers in Psychology, 1, 8.

Donohue, S. E., Darling, E. F., \& Mitroff, S. R. (2012b). Links between multisensory processing and autism. Experimental Brain Research. Experimentelle Hirnforschung. Experimentation Cerebrale, 222(4), 377-387.

Donohue, S. E., Woldorff, M. G., \& Mitroff, S. R. (2010). Video game players show more precise multisensory temporal processing abilities. Attention, Perception, \& Psychophysics, 72(4), 1120-1129.

Dye, M. W., Green, C. S., \& Bavelier, D. (2009a). Increasing speed of processing with action video games. Current Directions in Psychological Science: A Journal of the American Psychological Society, 18(6), 321-326.

Dye, M. W., Green, C. S., \& Bavelier, D. (2009b). The development of attention skills in action video game players. Neuropsychologia, 47(8-9), 1780-1789.

Feng, J., Spence, I., \& Pratt, J. (2007). Playing an action video game reduces gender differences in spatial cognition. Psychological Science, 18(10), 850-855.

Gold, J.I. and Ding, L. (2012) How mechanisms of perceptual decision-making affect the psychometric function. Progress in neurobiology.

Gold, J. I., \& Shadlen, M. N. (2007). The neural basis of decision making. Annual Review of Neuroscience, 30, 535-574.

Graziano, M., \& Sigman, M. (2008). The dynamics of sensory buffers: Geometric, spatial, and experience-dependent shaping of iconic memory. Journal of Vision, 8(5), 9. 1-13.

Green, C. S., \& Bavelier, D. (2003). Action video game modifies visual selective attention. Nature, 423(6939), 534-537.

Green, C. S., \& Bavelier, D. (2006a). Enumeration versus multiple object tracking: The case of action video game players. Cognition, 101(1), 217-245.

Green, C. S., \& Bavelier, D. (2006b). Effect of action video games on the spatial distribution of visuospatial attention. Journal of Experimental Psychology. Human Perception and Performance, 32(6), $1465-1478$.

Green, C. S., \& Bavelier, D. (2007). Action-video-game experience alters the spatial resolution of vision. Psychological Science, 18(1), 88-94.

Green, C. S., \& Bavelier, D. (2012). Learning, attentional control, and action video games. Current Biology: CB, 22(6), R197-R206.

Green, C. S., Pouget, A., \& Bavelier, D. (2010). Improved probabilistic inference as a general learning mechanism with action video games. Current Biology: CB, 20(17), 1573-1579.

Hubert-Wallander, B., Green, C. S., Sugarman, M., \& Bavelier, D. (2011). Changes in search rate but not in the dynamics of exogenous 
attention in action videogame players. Attention, Perception, \& Psychophysics, 73(8), 2399-2412.

Irons, J. L., Remington, R. W., \& McLean, J. P. (2011). Not so fast: Rethinking the effects of action video games on attentional capacity. Australian Journal of Psychology, 63(4), 224-231.

Karle, J. W., Watter, S., \& Shedden, J. M. (2010). Task switching in video game players: Benefits of selective attention but not resistance to proactive interference. Acta Psychologica, 134(1), 70-78.

Kristjansson, A. (2013). The case for causal influences of action videogame play upon vision and attention. Attention, perception $\&$ psychophysics.

Kuhbandner, C., Spitzer, B., \& Pekrun, R. (2011). Read-out of emotional information from iconic memory: The longevity of threatening stimuli. Psychological Science, 22(5), 695-700.

Li, R., Polat, U., Makous, W., \& Bavelier, D. (2009). Enhancing the contrast sensitivity function through action video game training. Nature Neuroscience, 12(5), 549-551.

Long, G. M. (1980). Iconic memory: A review and critique of the study of short-term visual storage. Psychological Bulletin, 88(3), 785-820.

Lu, Z. L., Neuse, J., Madigan, S., \& Dosher, B. A. (2005). Fast decay of iconic memory in observers with mild cognitive impairments. Proceedings of the National Academy of Sciences, USA, 102(5), 1797-1802.

Lucas, K., \& Sherry, J. L. (2004). Sex differences in video game play: A communication-based explanation. Communication Research, 31(5), 499-523.

Mandler, G., \& Shebo, B. J. (1982). Subitizing: An analysis of its component processes. Journal of Experimental Psychology. General, 111(1), 1-22.

Neisser, U. (1967). Cognitive psychology. Prentice Hall: Englewood Cliffs.

Nikolić, D., Häusler, S., Singer, W., \& Maass, W. (2009). Distributed fading memory for stimulus properties in the primary visual cortex. PLoS Biology, 7(12), e1000260.

Okagaki, L., \& Frensch, P. A. (1994). Effects of video game playing on measures of spatial performance: Gender effects in late adolescence. Journal of Applied Developmental Psychology, 15(1), 3358.

Orosy-Filders, C., \& Allan, R. W. (1989). Psychology of computer use: XII. Video-game play - human reaction-time to visual-stimuli. Perceptual and Motor Skills, 69(1), 243-247.
Persuh, M., Genzer, B., \& Melara, R. D. (2012). Iconic memory requires attention. Frontiers in Human Neuroscience, 6, 126.

Quaiser-Pohl, C., Geiser, C., \& Lehmann, W. (2006). The relationship between computergame preference, gender, and mentalrotation ability. Personality and Individual Differences, 44(3), 609-619.

Ratcliff, R., \& Smith, P. L. (2004). A comparison of sequential sampling models for two-choice reaction time. Psychological Review, $111(2), 333-367$.

Ruff, C. C., Kristjansson, A., \& Driver, J. (2007). Readout from iconic memory and selective spatial attention involve similar neural processes. Psychological Science, 18(10), 901-909.

Sperling, G. (1960). The information available in brief visual presentations. Psychological Monographs: General and Applied, 74(11), 1-29.

Strobach, T., Frensch, P. A., \& Schubert, T. (2012). Video game practice optimizes executive control skills in dual-task and task switching situations. Acta Psychologica, 140(1), 13-24.

Sungur, H., \& Boduroglu, A. (2012). Action video game players form more detailed representation of objects. Acta Psychologica, 139(2), 327-334.

Terlecki, M. S., \& Newcombe, N. S. (2005). How important is the digital divide? The relation of computer and videogame usage to gender differences in mental rotation ability. Sex Roles, 53(5), 433-441.

Trick, L. M., Jaspers-Fayer, F., \& Sethi, N. (2005). Multiple-object tracking in children: The "catch the spies" task. Cognitive Development, 20, 373-387.

West, G. L., Stevens, S. A., Pun, C., \& Pratt, J. (2008). Visuospatial experience modulates attentional capture: Evidence from action video game players. Journal of Vision, 8(16, Art. 13), 1-9.

Wickens, T. D. (2001). Elementary signal detection theory. New York: Oxford University Press.

Wilms, I. L., Petersen, A., \& Vangkilde, S. (2013). Intensive video gaming improves encoding speed to visual short-term memory in young male adults. Acta Psychologica, 142(1), 108-118.

Wu, S., Cheng, C. K., Feng, J., D'Angelo, L., Alain, C., \& Spence, I. (2012). Playing a first-person shooter video game induces neuroplastic change. Journal of Cognitive Neuroscience, 24(6), $1286-1293$.

Yuji, H. (1996). Computer games and information-processing skills. Perceptual and Motor Skills, 83(2), 643-647. 